\title{
MIMiC: Multimodal Interactive Motion Controller
}

\author{
Dumebi Okwechime, Member, IEEE, Eng-Jon Ong, and Richard Bowden, Senior Member, IEEE
}

\begin{abstract}
We introduce a new algorithm for real-time interactive motion control and demonstrate its application to motion captured data, pre-recorded videos and HCI. Firstly, a data set of frames are projected into a lower dimensional space. An appearance model is learnt using a multivariate probability distribution. A novel approach to determining transition points is presented based on k-medoids, whereby appropriate points of intersection in the motion trajectory are derived as cluster centres. These points are used to segment the data into smaller subsequences. A transition matrix combined with a kernel density estimation is used to determine suitable transitions between the subsequences to develop novel motion. To facilitate real-time interactive control, conditional probabilities are used to derive motion given user commands. The user commands can come from any modality including auditory, touch and gesture. The system is also extended to HCI using audio signals of speech in a conversation to trigger non-verbal responses from a synthetic listener in real-time. We demonstrate the flexibility of the model by presenting results ranging from data sets composed of vectorised images, $2 D$ and 3D point representations. Results show real-time interaction and plausible motion generation between different types of movement.
\end{abstract}

Index Terms-Human computer interaction, motion, probability density function, animation, synthesis.

\section{INTRODUCTION}

$\mathbf{M}$ OTION synthesis has extensive applications and has been a challenging topic of research for many years. The human visual system has the ability to efficiently and easily recognise characteristic motion especially human movement. As a consequence, in order to generate animations that look realistic, it is necessary to develop methods to capture, maintain and synthesise intrinsic style to give authentic realism to motion data. Likewise, when filming a movie, certain elements in a video scene such as the movement of trees blowing in the wind, do not perform on cue. It may not always be cost effective, safe or even possible to control the surroundings to match the director's intentions. These issues are addressed in this paper. By modelling the motion as a pose space Probability Density Function (PDF) and using a Markov Transition Matrix to apply additional constraints to the motion dynamics, a Motion Model is developed that can synthesise novel sequences in real time whilst retaining the natural variances inherit to the original data. Additionally, by learning the mapping between motion subspaces and external stimulus, the user can drive the motion at an intuitive level, giving the user real-time interactive Multimodal Control of the

Manuscript received April 29, 2010; revised November 24, 2010. This work was supported by the EPSRC project LILiR (EP/E027946/1) and the European Community's Seventh Framework Programme (FP7/2007-2013) under grant agreement number 231135 - DictaSign.

The authors are with the Department of CVSSP, University of Surrey, Guildford, Surrey, GU2 7XH, UK.

E-mail: \{d.okwechime,e.ong,r.bowden\}@surrey.ac.uk.

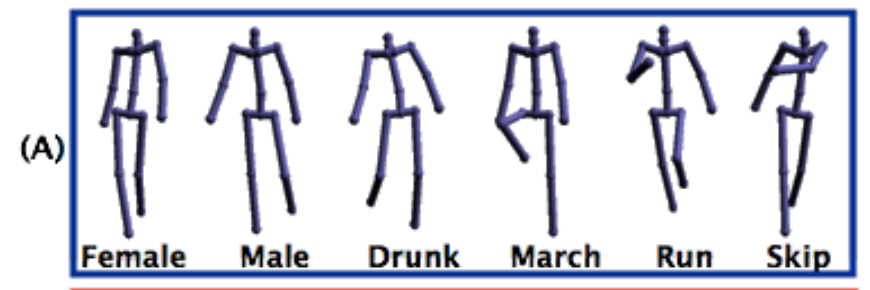

(B)

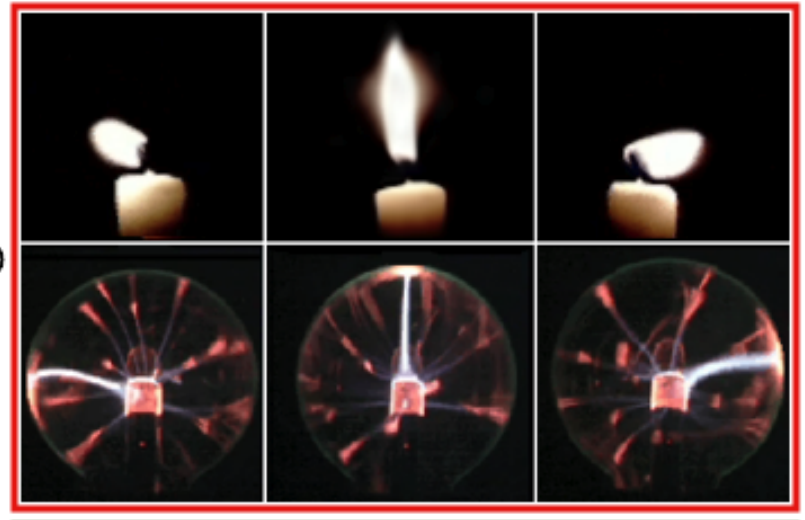

(C)

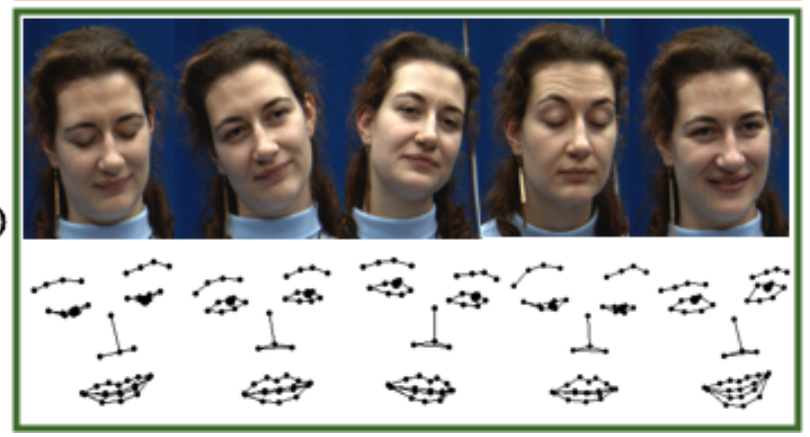

Fig. 1. (A) Sample motion captured data of different types of walks, (B) Candle and plasma beam recorded whilst undergoing motion, (C) Tracked face data used in modelling conversational cues.

creation of novel sequences. The external stimulus could come from any modality and we demonstrate the use of auditory, touch and gesture within this work. Combining the real-time Motion Model with interactive Multimodal Control, we get the Multimodal Interactive Motion Controller (MIMiC), giving a user multimodal control of motion data of various formats.

Figure 1 shows the example applications. Figure 1 (A) shows six different types of motion captured walks. Using MIMiC, we are able to generate novel movement and transitions between different types of cyclic motion such as running and skipping. Figure 1 (B) shows example frames from video sequences of a candle flame and plasma ball used as video textures. Here, the purpose of MIMiC is to control the direction in which the flame and beam move in real-time, whilst generating a novel animation with plausible transitions between different types of movement. Figure 1 (C) shows a 2D 
tracked contour of a face generated from a video sequence of a person listening to a speaker. Mapping the audio features of the speaker to the 2D face, we generate appropriate non-verbal responses triggered by audio input.

The paper is divided into the following sections. Section II, briefly details related works in the field of motion texture synthesis, and HCI. Section III presents an overview of the MIMiC system. Sections IV and V detail the techniques used in data representation and dimension reduction respectively. Sections VI and VII describe the process of learning the motion model and synthesising novel motion sequences. Section VIII presents the interactive multimodal controller, and the remainder of the paper describes the results and conclusions.

\section{RELATED WORK}

Synthesis has extensive applications in graphics and computer vision, and can be categorised into three groups: texture synthesis of discrete images, temporal texture synthesis in videos, and motion synthesis in motion captured data. Early approaches to texture synthesis were based on parametric [1] [2] and non-parametric [3] [4] methods, which create novel textures from example inputs. Kwatra et al [5] generate perceptually similar patterns from a small training data set, using a graph cut technique based on Markov Random Fields (MRF). Approaches to static texture synthesis have paved the way for temporal texture synthesis methods, often used in the movie and gaming industries for animating photorealistic characters and editing video scenery. An example is presented by Bhat et al [6] who used texture particles to capture dynamics and texture variation travelling along user defined flow lines. This was used to edit dynamic textures in video scenery.

In some cases, techniques used for the synthesis of motion captured data are similar to the techniques used for temporal texture synthesis of videos. By substituting pixel intensities (or other texture features) with marker co-ordinates, and applying motion constraints suited to the desired output, a similar framework can be extended to both domains.

A number of researchers have used statistical models to learn generalised motion characteristics for the synthesis of novel motion. Troje [7] used simple sine functions to model walking and running motions. Pullen and Bregler [8] used a kernel based probability distribution to extract a 'motion texture' (i.e. the personality and realism, from the motion capture data) to synthesize novel motion with the same style and realism of the original data. Okwechime and Bowden [9] extended this work using a multivariate probability distribution to blend between different types of cyclic motion to create novel movement. Wang et al [10] proposed a non-parametric dynamical system based on a Gaussian processes latent variable model, which learns a representation for a nonlinear system. All these systems use a generalisation of the motion rather than the original data, and cannot guarantee that the synthesised motion is physically realistic or looks natural.

Motion synthesis using example-based methods, i.e. retaining the original motion data to use in synthesis, provides an attractive alternative as there is no loss of detail from the original data. Tanco and Hilton [11] presented a twolevel statistical model, based on a Markov chain and a Hidden Markov Model, for modelling skeletal motion captured data, that derive optimal sequences between user defined key-frames. Representing motion transitions using a motion graph [12][13][14][15], originally introduced by Kovar et al [16], provides additional user-control on positioning, using sequences from the original data and automatically generated transitions to perform an optimal graph walk that satisfies userdefined constraints. Our method expands on this, using a pose space PDF to derive the likelihood of a pose given the data, ensuring better quality transitions. Also, these methods are tailored to motion captured data, whereas our motion model is generic to data formats, applicable to both motion capture and dynamic textures in video.

Arikan et al [17] allow users to synthesize motion by creating a timeline with annotated instructions such as walk, run or jump. Treuille et al [18] developed a system that synthesizes kinematic controllers which blend subsequences of precaptured motion clips to achieve a desired animation in real-time. The limitation to this approach is it requires manual segmentation of motion subsequences to a rigid design in order to define appropriate transition points. Our system uses an unsupervised k-medoid algorithm to derive appropriate transition points automatically.

Intuitive interfaces to control motion data are difficult because motion data is intrinsically high dimensional and most input devices do not map well into this space. Mouse and keyboard interfaces can only give position and action commands, so an autonomous approach is needed to translate user commands to appropriate behaviours and transitions in modelled motion data. Schödl et al [19] introduced Video Textures which generates a continuous stream of video images from a small amount of training video. Their system was demonstrated on several examples including a mouse controlled fish, whereby a mouse cursor was used to guide the path of the fish with different velocities. Similarly, Flagg et al [20] presented Human Video Textures, where, given a video of a martial artist performing various actions, they produce a photorealistic avatar which can be controlled, akin to a combat game character. Lee et al [21] used interactive controllers to animate an avatar from human motion captured data. They present three control interfaces: selecting a path from available choices to control the motion of the avatar, manually sketching a path (analogous to Motion Graphs [16]), and acting out motion in front of a camera for the avatar to perform. Our multimodal controller is demonstrated on a keyboard, mouse interface and on vision methods, such as performing gestures in front of a camera. We also extend our controller to the audio domain, using audio MFCC features to drive the motion model.

Previous approaches to modelling motion driven by audio features, have been used for lip-syncing a facial model [22] [23], or animating the hand and body gestures of a virtual avatar [24]. In these examples, audio signals are used to animate a speaker or performer. Jebara and Pentland [25] touched on modelling conversational cues and proposed Action Reaction Learning (ARL), a system that generates an animation of appropriate hand and head pose in response to a users 


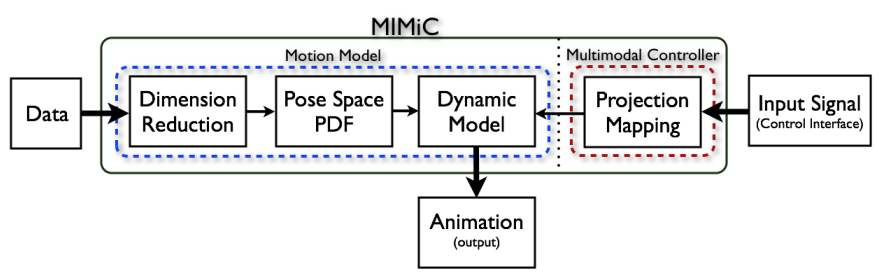

Fig. 2. Flow chart of MIMiC system. Consists of two main stages, the Motion Model and the Multimodal Controller. The Motion Model takes a data set and creates a dynamic model of motion. The Multimodal Controller uses projection mapping to translate user commands from an input signal to the dynamic model. The system generates the desired output as synthesised novel animations

hand and head movement in real-time. However, this does not incorporate audio. In this paper, we demonstrate the flexibility of the multimodal controller, modelling conversational cues based on audio input. Audio features of the speaker are used to derive appropriate visual responses of the listener in real-time from a 2D face contour modelled with the motion controller. This results in plausible head movement and facial expressions in response to the speaker's audio.

A preliminary version of this work appeared in [26]. This extended manuscript presents the full MIMiC system with additional formalisation and adds further evaluation data sets demonstrating the applicability of the approach to the synthesis of Motion capture data, video texture synthesis and human computer interaction. Furthermore, we demonstrate a range of input modalities from keyboard and mouse events through to computer vision and speech.

\section{OVERVIEW}

MIMiC allows a user to reproduce motion in a novel way by specifying, in real-time, which type of motion inherent in the original sequence to perform. As shown in Figure 2, the system comprises two stages: learning a Motion Model, and building a Multimodal Controller.

The process of learning a Motion Model starts with the data, which is the input to the system. The data can be of various formats (see Section IV). Given the data, eigenspace decomposition is used to reduce the dimensionality to a lower dimensional space which we refer to as pose space. Using kernel density estimation, a pose space PDF is learnt. A fast approximation method based on kd-trees is proposed to speed up this estimation process for real time execution. An unsupervised segmentation method derives cut point clusters, whereby each cluster represents groups of similar frames that can be seamlessly blended together. These cut points are used as transition points, through which the set of consecutive frames between adjoined transition points make up subsequences. A first-order Markov Transition Matrix is learnt by treating each cut point cluster as a state in a Markov process. Motion is generated as high likelihood transitions from one subsequence to another based on the pose space PDF and the probability of the given transition determined by the Markov Transition Matrix.

The second stage is the Multimodal Controller which allows real-time manipulation of the Motion Model based upon an input signal. The controller consists of a projection mapping between the model and input signal, which reweights the pose space PDF to produce the desired movement.

\section{DATA}

Given a motion sequence $\mathbf{X}$, each frame is represented as a vector $\mathbf{x}_{i}$ where $\mathbf{X}=\left\{\mathbf{x}_{1}, \ldots, \mathbf{x}_{N_{T}}\right\}$ and $N_{T}$ is the number of frames.

Various motions can be modelled by the system. We demonstrate 3D motion, 2D tracked points, and rgb pixel intensities in four examples:

- 3D Motion Captured Data: The user can specify in real-time which type of animated walk to generate. By requesting a set of different walks, the system can blend between them whilst retaining the natural variance inherent in the original data. Six different walks are used: male walk, female walk, drunk walk, march, run, and skip.

- Candle Flame: We synthesise the movement of a candle flame where the user has control over three discrete states: ambient flame, flame blow left, flame blow right, and can blend between them. Using simple computer vision, the user can perform hand-waving gestures to influence the direction of the flame, giving the illusion of creating a draft/breeze that influences the animation.

- Plasma Beam: The user controls the movement of a plasma beam using a mouse cursor or a touch screen monitor. The plasma beam responds to the user's touch in real-time.

- Tracked 2D Face Contour: An animation of a 2D face is driven directly from audio speech signals, displaying appropriate non-verbal visual responses for an avid listener based on a speakers audio signal.

In all cases, each time step $i$ of the data to be modelled is vectorised as $\mathbf{x}_{i}=\left(x_{i 1}, y_{i 1}, \ldots, x_{i a}, y_{i a}\right) \in \Re^{2 a}$ for a $2 \mathrm{D}$ contour of $a$ points, $\mathbf{x}_{i}=\left(x_{i 1}, y_{i 1}, z_{i 1}, \ldots, x_{i b}, y_{i b}, z_{i b}\right) \in$ $\Re^{3 b}$ for a 3D contour of $b$ points and $\mathbf{x}_{i}=$ $\left(r_{11}, g_{11}, b_{11}, \ldots, r_{\mathrm{xy}}, g_{\mathrm{xy}}, b_{\mathrm{xy}}\right) \in \Re^{\mathrm{xy}}$ for an $\mathrm{x} \times \mathrm{y}$ image

\section{Dimension Reduction}

To reduce the complexity of building a generative model of motion, Principal Component Analysis (PCA) [27] [28] is used for dimensionality reduction. Since the dimensionality of the resulting space does not necessarily reflect the true dimensionality of the subspace the data occupies, only a subset of the eigenvectors are required to accurately model the motion.

The dimension of the feature space $\left|\mathbf{x}_{i}\right|$ is reduced by projecting into the eigenspace

$$
\mathbf{y}_{i}=\mathbf{V}^{T}\left(\mathbf{x}_{i}-\boldsymbol{\mu}\right)
$$

where $\mathbf{V}$ is the projection onto the eigenspace $\mathbf{V}=\left[\mathbf{T}_{1}, \ldots, \mathbf{T}_{d}\right]$, $\mathbf{T}_{i}$ are the eigenvectors, $\boldsymbol{\lambda}_{i}$ the eigenvalues, $\boldsymbol{\mu}$ is the sample mean $\boldsymbol{\mu}=\frac{1}{N_{T}} \sum_{i=1}^{N_{T}} \mathbf{x}_{i}$, and $d$ is the chosen lower dimension $d \leq\left|\mathbf{x}_{i}\right|$ such that $\sum_{i=1}^{d} \frac{\lambda_{i}}{\Sigma \forall \lambda} \geq .95$ or $95 \%$ of the energy is retained. $\mathbf{Y}$ is defined as a set of all points in the dimensionally reduced data where $\mathbf{Y}=\left\{\mathbf{y}_{1}, \ldots, \mathbf{y}_{N_{T}}\right\}$ and $\mathbf{y}_{i} \in \Re^{d}$. This results in a $d$-dimensional representation of each frame in the 

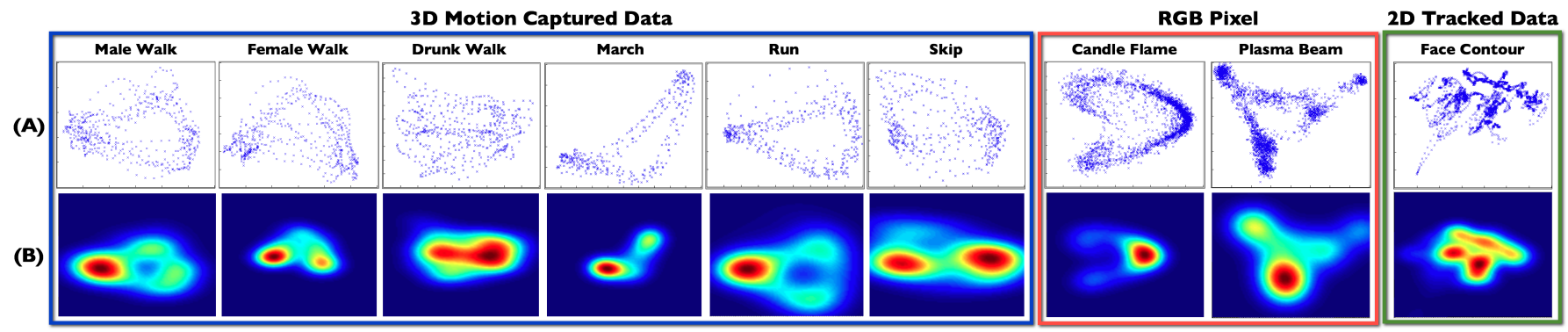

Fig. 3. Row (A): Plot of eigen projections of the first 2 dimensions of all data sets. Row (B): PDF of pose space where the kernel size has been scaled by $\alpha=0.25$

sequence. This representation reduces the computational and storage complexity of the data whilst still retaining the time varying relationships between each frame.

Figure 3 row (A) shows plots of the different data sets projected onto the first two eigenvectors. They produce a nonlinear but continuous subspace characteristic of continuous motion, showing that the projection retains the non-linearity of the movement.

\section{Pose Space PDF}

Conventional motion graph synthesis traverses the graph, connecting motion segments based on user specified constraints such as position, orientation and timing $[12][13][14][15][16]$. Little interest is given to how common or likely the connecting nodes are given the data set. Better quality transitions can be produced by computing the likelihood of a pose or frame as an additional parameterised weight. Hence, a dynamic model is learnt to derive the likelihood of pose and motion in eigenspace based on the respective data set.

A statistical model of the constraints and dynamics present within the data can be created using a PDF. A PDF of appearance is created using kernel estimation where each kernel $p\left(\mathbf{y}_{i}\right)$ is effectively a Gaussian centred on a data example $p\left(\mathbf{y}_{i}\right)=G\left(\mathbf{y}_{i}, \Sigma\right)$. Since we want our probability distribution to represent the dimensionally reduced data set $\mathbf{Y}$ as noted in Section V, the likelihood of a pose in pose space is modelled as a mixture of Gaussians using multivariate normal distributions. We will refer to this Gaussian mixture model as the pose space PDF.

$$
P(\mathbf{y})=\frac{1}{N_{T}} \sum_{i=1}^{N_{T}} p\left(\mathbf{y}_{i}\right)
$$

where the covariance of the Gaussian is:

$$
\Sigma=\alpha\left(\begin{array}{ccc}
\sqrt{\lambda_{1}} & \cdots & 0 \\
\vdots & \ddots & \vdots \\
0 & \cdots & \sqrt{\lambda_{d}}
\end{array}\right)
$$

Figure 3 row (B) shows a plot of such a distribution for each data set with the first mode plotted against the second mode. The width of the Gaussian in the $i^{\text {th }}$ dimension is set to $\alpha \sqrt{\lambda_{i}}$. If $\alpha=0$, i.e. the variance is set to 0 , the synthesis will not generalise and simply replay the original data. If $\alpha$ is too high, there is no constraint upon pose and the resulting animation will be destroyed. As the eigenvalues are based on the variance of the overall data set, this allows the PDF to scale appropriately to the data. Therefore, we chose $\alpha$ experimentally to provide a good trade off between accurate representation and generalisation, but it is important to note that this parameter remains fixed for all data sets. For all experiments $\alpha=0.25$.

\section{A. Fast Gaussian Approximation}

As can be seen from Equation 2, the computation required for the probability density estimation is high since it requires an exhaustive calculation from the entire set of data examples. This would be too slow for a real time implementation. The more samples used, the slower the computation, however, the more accurate the density estimation. As a result, a fast approximation method based on kd-trees [29] is used to reduce the estimation time without sacrificing accuracy.

Instead of computing kernel estimations based on all data points, with the kd-tree we can localise our query to neighbouring kernels, assuming the kernel estimations outside a local region contribute nominally to the local density estimation. We are now able to specify $N_{n}$ nearest neighbours to represent the model, where $N_{n}<N_{T}$. This significantly reduces the amount of computation required.

Equation 2 is simplified to:

$$
P^{\prime}(\mathbf{y})=\frac{1}{\left|\mathbf{Y}^{\prime}\right|} \sum_{\forall \mathbf{y}_{i} \in \mathbf{Y}^{\prime}} p\left(\mathbf{y}_{i}\right)
$$

where $\mathbf{Y}^{\prime} \subseteq \mathbf{Y}$, and $\mathbf{Y}^{\prime}$ is a set containing the $N_{n}$ nearest neighbouring kernels to $\mathbf{y}$ found efficiently with the kd-tree.

\section{DyNAMiC MODEL}

By learning a PDF, the data is represented in a generalised form which is analogous to a generative model. Using this form on its own, it is possible to generate novel motion, using pre-computed motion derivatives, combined with a gradient decent for optimisation. However, such a model runs the risk of smoothing out subtle motion details, and is only suited for simple motion. To overcome these limitations, we segment the original data into shorter subsequences, and combine the PDF with a Markov Transition Matrix to determine the likelihood of transitioning to a subsequence given a pose configuration. This 


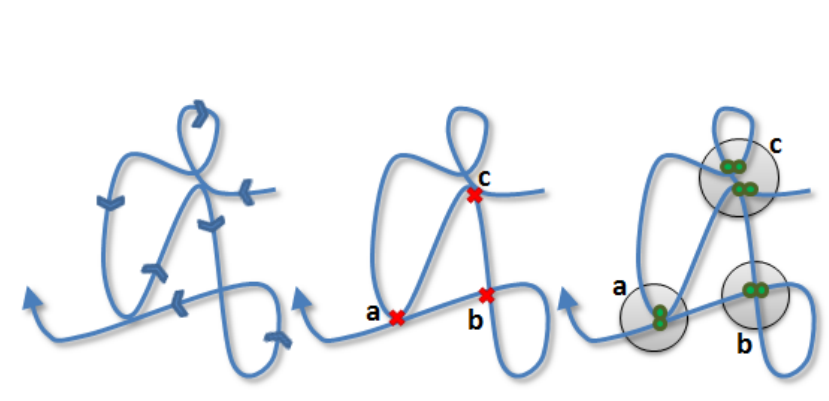

(A)
(B)

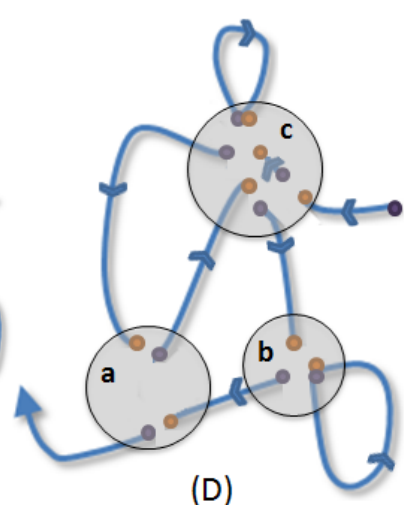

(D)

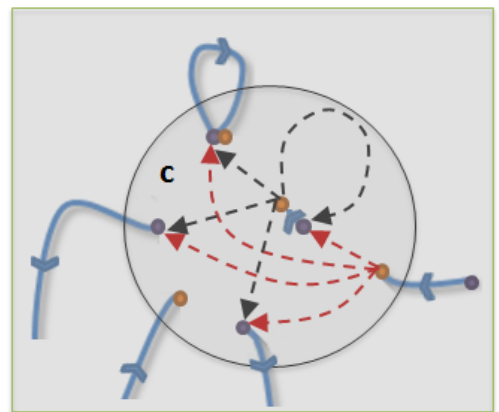

(E)

Fig. 4. (A) Trajectory of the original motion sequence. Arrows indicate the direction of motion. (B) $N_{c}=3 \mathrm{k}$-medoid points derived using the unsupervised k-medoid clustering algorithm. The three red crosses are the three k-medoid points a, b, and c. (C) The small green dots are the cut points derived as the nearest neighbouring points to a k-medoid point less than a user defined threshold $\theta$. The three gray circles represent cut point clusters a, b and c. (D) Cut points act as start and end transition points segmenting the data into shorter segments. The orange dots are start transition points and the purple dots are end transition points. (E) Diagram of possible transitions within cluster c. For simplicity only a few transitions are displayed.

allows motion generation based on the original data, retaining subtle but important motion information. It also allows our motion model to work with non-periodic motion data.

The reminder of this Section is divided into four parts. First, we describe our unsupervised segmentation approach. In the following three Sections, we explain our Markov Transition Matrix, how we generate novel motion sequences, and our dynamic programming method for forward planning.

\section{A. Unsupervised Motion Segmentation}

Similar to most work on motion synthesis, the motion data needs to be analysed to compute some measure of similarity between frames and derive points of intersection within the data. These points are used to segment the motion data into several short subsequences, where a single subsequence is represented as a set of consecutive frames between a start and end transition point. The idea is to connect various subsequences together to create a plausible novel sequence.

The common approach is to compute the L2 distance over a window of frames in time and use a user defined threshold to derive points of intersection within the data to use as transition points [16][20][19][12][13]. This approach works well, however, for large data sets, it can be tedious to compute the distance between every frame. Balci et al [14] proposed an iterative clustering procedure based on k-means to define clusters of poses suitable for transitions. However, k-means produces cluster centres not embedded in the data which can result in noise and outliers. Instead, we adopt a kmedoid cluster algorithm to define $N_{c}$ k-medoid points, where $N_{c}<N_{T}$. Each k-medoid point is defined as the local median in regions of high density, and can be used to define regions where appropriate transitions are possible. By only computing the L2 distance at these points, we reduce the amount of computation required to define candidate transitions, focusing attention on regions where transitions are most likely.

Figure 4 shows an example of the process. Given a motion sample, shown by the two dimensional motion trajectory in Figure 4 (A), a k-medoid clustering algorithm is used to find
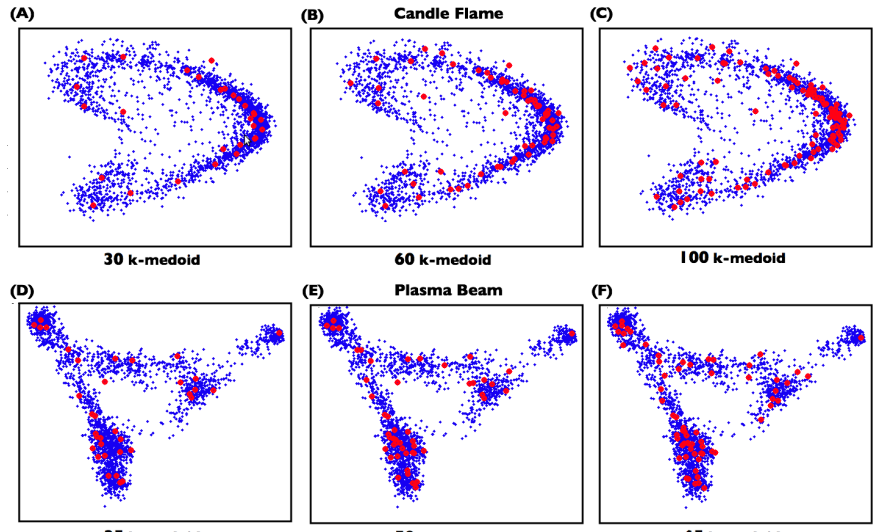

Fig. 5. Plot showing the distributions of varying numbers of k-medoids relative to the data set. (A), (B), and (C) relate to the candle flame data set, and (D), (E), and (F) to the plasma beam data set. The blue points are the eigen projections of the first 2 principal components, and the red points are the k-medoids.

$N_{c}$ k-medoid points. We define each k-medoid point as $\boldsymbol{\delta}_{n}^{c}$ given by the k-medoid method whereby $\boldsymbol{\delta}_{n}^{c} \in \mathbf{Y}$. In Figure 4 (B), $N_{c}=3$ and are shown as the three red crosses which we refer to as $a, b$, and $c$.

$N_{c}$ is empirically determined based on the number of clusters that best defines the distribution of poses in pose space. This is demonstrated in Figure 5, showing the distributions of varying numbers of k-medoids relative to the data set. Figure 5(A) and (D) shows a low distribution of 30 and $35 \mathrm{k}$ medoid points for the candle flame and plasma beam data set respectively. Though the most densely populated areas have sufficient distribution of k-medoids points, the less densely populated areas do not. As a result, $N_{c}$ is increased until a satisfactory distribution in pose space has been reached. Shown in Figure 5(C) and (F), a high distribution of 100 and $65 \mathrm{k}$-mediods for the candle flame and plasma beam data set respectively, present a better spread of k-medoids across the respective data sets.

The outcome of varying this parameter is qualitative. $N_{c}$ 

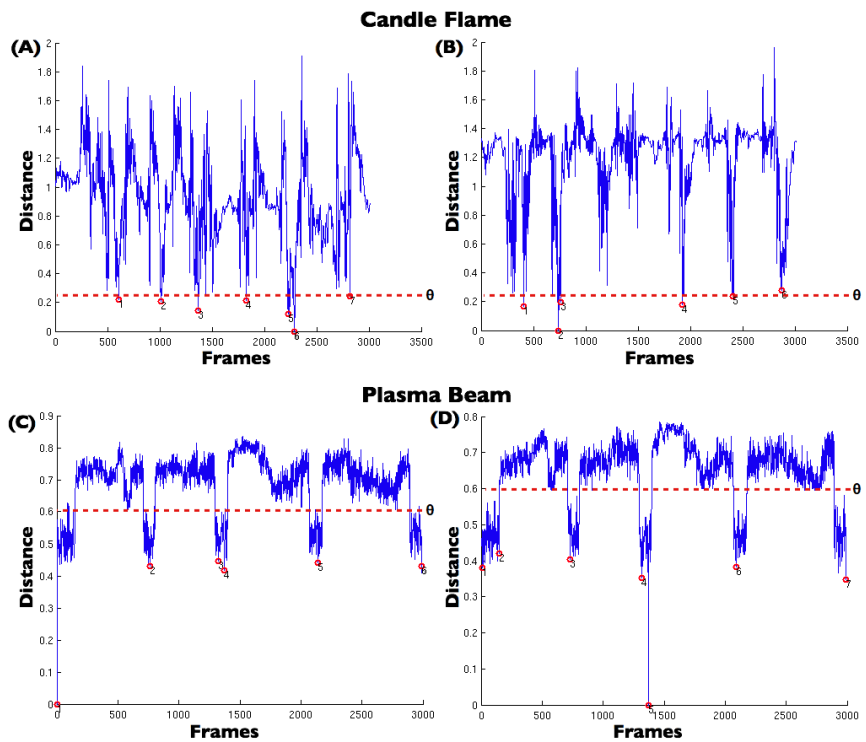

Fig. 6. Plot showing the $\mathrm{L} 2$ distance between two different k-medoid points and all points in the data set, for the candle flame data set ((A) and (B)), and the plasma beam data set ((C) and (D))

is not sensitive to small variations, having a large range over which it makes little difference to animation. However, if $N_{c}$ is too high, the model will generate unrealistic motion as a result of shorter motion subsequences causing highly frequent and unnatural transitions. If $N_{c}$ is too low, the subsequences will be too long, reducing the novelty of animation and the model's responsiveness to user commands. Different values of $N_{c}$ were chosen for the different data sets based on this condition, and are detailed in Section IX.

Using a user defined threshold $\theta$, the nearest points to each k-medoid points are identified to form clusters of cut points. The cut points are represented by the small green dots in Figure 4 (C), and the clusters of cut points are represented by the gray circles. The set containing the cut points of the $n^{t h}$ cluster is defined as $\mathbf{Y}_{n}^{c}=\left\{\mathbf{y}_{n, 1}^{c}, \ldots, \mathbf{y}_{n, Q_{n}}^{c}\right\}$, where the number of cut points of the $n^{\text {th }}$ cluster is denoted as $Q_{n}$.

Threshold $\theta$ provides the user with a tolerance on how close cut points need to be in eigenspace to form a valid transition. It is determined experimentally whereby if it is set too high, it becomes more challenging to produce plausible blends when making transitions, and if too low, potential cut points are ignored and we are limited to points that overlap, which is an unlikely occurance in a multi-dimensional space. This is demonstrated in Figure 6. The graphs show the L2 distance between a k-mediod point and all points in the data set. There are two examples for the candle flame data set (Figure 6 (A) and (B)), and the plasma beam data set (Figure 6 (C) and (D)). The red line across the graph represents the chosen threshold $\theta$. The value of $\theta$ for each data set (shown in Section IX) where chosen to set an acceptable trade-off between having good transitions (low threshold) and having high connectivity (high threshold).

The cut point clusters consist of discrete frames which are not directly linked, however smooth transitions can be

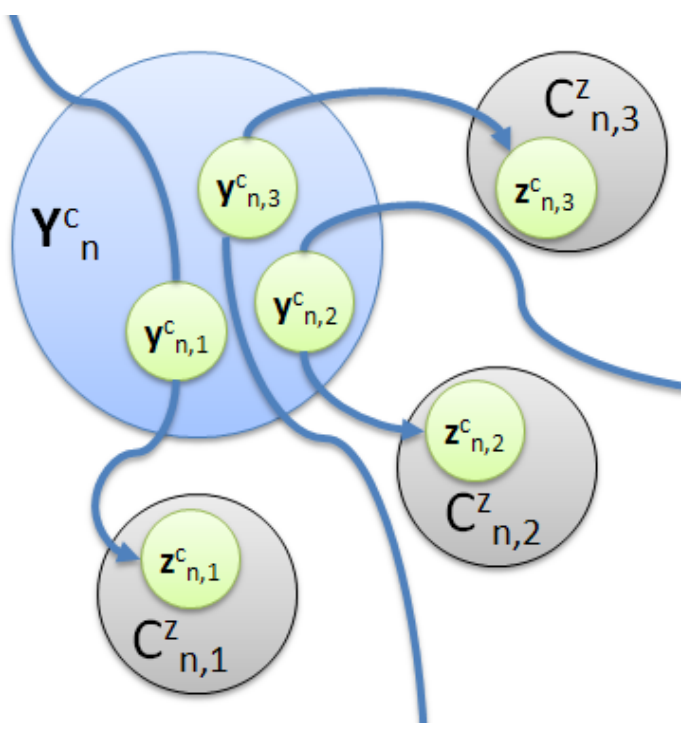

Fig. 7. Example of transitions between cut points in different clusters. The six green circles are cut points and the blue lines are sets of consecutive frames connecting them. These sets of consecutive frames make up the different subsequences. The blue circle $\mathbf{Y}_{n}^{c}$, represents the cluster of start transition points, and the three grey circles indexed as $C_{n}^{z}$, represents the clusters of end transition points

made between them. Simple blending techniques such as linear interpolation can reliably generate a transition. The simplicity of linear interpolation also allows for quick computation, supporting real-time animation rendering during motion blending. As shown in Figure 4 (D) the cut points are used to segment the data to smaller subsequences with start and end transition points. Figure 4 (E) shows a few of the possible transitions between various subsequences in cluster $\mathrm{c}$.

In cases where the recovered cut point clusters in pose space are sparsely populated, they are automatically pruned and removed from the network of clusters.

Shown in Figure 7, for simplicity, we define the transitions from the $n^{\text {th }}$ cluster contents as the triplets $\left\{\left(\mathbf{y}_{n, 1}^{c}, \mathbf{z}_{n, 1}^{c}, C_{n, 1}^{z}\right), \ldots,\left(\mathbf{y}_{n, Q_{n}}^{c}, \mathbf{z}_{n, Q_{n}}^{c}, C_{n, Q_{n}}^{z}\right)\right\}$, where $\mathbf{y}_{n}^{c}$ is a cut point in the $n^{\text {th }}$ cluster acting as the start transition point, $\mathbf{z}_{n}^{c}$ is the end transition point denoting the end of the subsequence between $\mathbf{y}_{n}^{c}$ and $\mathbf{z}_{n}^{c}$, where $\mathbf{z}_{n}^{c} \in \mathbf{Y}^{c}$ and $\mathbf{z}_{n}^{c} \neq \mathbf{y}_{n}^{c}$, and $C_{n}^{z}$ is the index of the cluster $\mathbf{z}_{n}^{c}$ belongs to. In this example, $Q_{n}=3$.

In most of our data sets, there are variable densities across different motion types. A specific example is the candle flame data set which has a heavy bias towards the stationary flame state due to the quantity of data acquired for each state. This is evident in Figure 3 on the $7^{\text {th }}$ column. The $\mathrm{k}$-medoid algorithm attempts to find exemplars which cover the entire manifold/subspace of data points. Adding more of the uncommon motion types/animation to the data set is also possible as k-medoid will attempt to evenly partition the entire data set.

\section{B. Markov Transition Matrix}

When generating novel motion sequences, we are not only interested in generating the most likely pose but also the 
mostly probable path leading to it. Given that our eigenspace of data points are finite, a first order Markov Transition Matrix [30] is used to discourage movements that are not inherent in the training data. As an approach formally used with time-homogeneous Markov chains to define transition between states, by treating our clusters of cut points $\mathbf{Y}_{i}^{c}$ as states, this approach can be used to apply further constraints and increase the accuracy of the transition between sequences.

We define $\mathbf{P}=\left\{p_{k, l}\right\}$ as the transition matrix whereby $p_{k, l}$ denotes the probability of going from cluster $k$ to cluster $l$, and $\sum_{l} p_{k, l}=1$ learnt from the training data. We are now able to represent the conditional probability of moving from one cluster to another as $P\left(C_{t} \mid C_{t-1}\right)=p_{C_{t-1}, C_{t}}$ where $C_{t}$ is defined as the index for a cluster/state at time $t$ (where $t$ is in unit of frames). This transition matrix is constructed using the cut points within the sequence to identify the start and end transitions within the data.

The transition probability acts as a weighting, giving higher likelihood to transitions that occur more frequently in the original data. To account for situations where a transition might have zero probability, a nominal value is added to all elements in the transition matrix before normalisation. This allows the transition model to move between states not represented as transitions in the original sequence.

\section{Generating Novel Sequences}

To generate novel motion sequences the procedure is:

1) Given the current position in pose space $\mathbf{y}_{t}^{c}$, find all adjacent cut point neighbours in $\mathbf{Y}_{t}^{c}$ as defined in Section VII-A, to represent start transition points.

2) Find all associated end transition points $\mathbf{z}_{t, m}^{c} \mid m=$ $\left\{1, \ldots, Q_{t}\right\}$. This gives a set of $Q_{t}$ possible transitions from the starting point $\mathbf{y}_{t}^{c}$ in pose space.

3) Denote the cut point group index that $\mathbf{y}_{t}^{c}$ belongs to as $C_{t}$.

4) Calculate the likelihood of each transition as:

$$
\phi_{m}=P\left(C_{C_{t+1}, m}^{z} \mid C_{t}\right) P^{\prime}\left(\mathbf{z}_{C_{t+1}, m}^{c}\right)
$$

where $\boldsymbol{\Phi}=\left\{\phi_{1}, . ., \phi_{Q_{t}}\right\}$.

5) Normalise the likelihoods such that $\sum_{i=1}^{Q_{t}} \phi_{i}=1$.

6) Since a maximum likelihood approach will result in repetitive animations, we randomly select a new start transition point $\mathbf{y}_{t, k}^{c}$ from $\boldsymbol{\Phi}$ based upon its likelihood as:

$$
\underset{k}{\arg \min }\left(\sum_{j=1}^{k} \phi_{j} \geq r\right)
$$

where $k$ is the index of the newly chosen end transition point, $k \in m$, and $r$ is a random number between 0 and $1, r \in[0,1)$.

7) If $\mathbf{y}_{t}^{c} \neq \mathbf{y}_{t, k}^{c}$, use linear interpolation to blend $\mathbf{y}_{t}^{c}$ to $\mathbf{y}_{t, k}^{c}$ and reconstruct for rendering:

$$
\mathbf{x}_{\text {Lin }}=\mu+\mathbf{V}\left(\alpha(t) \mathbf{y}_{t}^{c}+[1-\alpha(t)] \mathbf{y}_{t, k}^{c}\right)
$$

8) All frames associated to the transition sequence between $\mathbf{y}_{t, k}^{c}$ and $\mathbf{z}_{t, k}^{c}$ are reconstructed for rendering as:

$$
\mathbf{x}_{t}=\left(\mu+\mathbf{V} \mathbf{y}_{t}\right)
$$

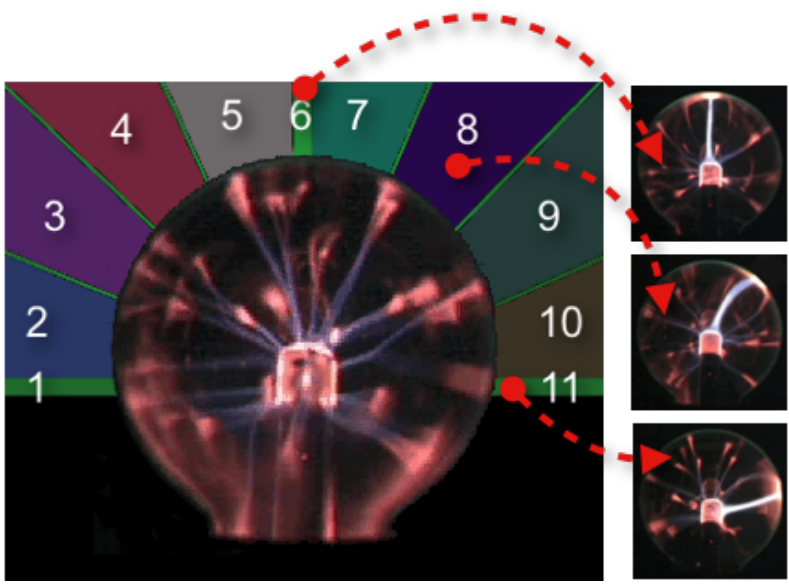

Fig. 8. Image showing the quantisation of the plasma beam into $N_{s}=11$ symbols, relating to the direction the plasma beam can be summoned

9) The process then repeats from step (1) where $\mathbf{y}_{t+1}^{c}=$ $\mathbf{z}_{t, k}^{c}$.

\section{Dynamic Programming}

In most cases, motion requires sacrificing short term objectives for the longer term goal of producing a smooth and realistic sequence. As a result, dynamic programming is used for forward planning. Formally used in Hidden Markov Models to determine the most likely sequence of hidden states, it is applied to the pose space PDF to observe the likelihoods for $m=3$ steps in the future.

Rendering speed of approximately 25 frames per second was obtained when using a 3 level trellis $m=3 . m$ is selected as the maximum number of trellis levels that allows real-time computation and animation rendering. For all data sets, $m>3$ resulted in no noticeable improvement in the quality of the synthesised animations, however, greatly reduced rendering speed.

Treating our clusters as states, a trellis is built $m$ steps in the future effectively predicting all possible transitions $m$ levels ahead. Dynamic programming is then used to find the most probable path for animation. Though it may take slightly longer to generate a desired motion, the overall result is more realistic. With this approach, we can also avoid potential 'deadends', which limit the types of motions that can be generated by the model.

\section{Multimodal Controller}

Thus far, the Motion Model randomly generates the mostly likely set of motion sequences given a starting configuration. To allow real-time control we introduce our Multimodal Controller, which uses a conditional probability to map between input space and pose space. This section describes the Projection Mapping method used in controlling the Motion Model.

\section{A. Projection Mapping}

The mapping is used when wanting to enable motion control of the generated motion. Firstly, the input space is 
quantised into an appropriate number of symbols $N_{s}$. These symbols are then associated to a set of training examples $E_{r}=e_{i} \mid i=\left\{1, \ldots, N_{r}\right\}$, where $N_{r}$ is the number of training examples associated to the $r^{t h}$ symbol, and $e_{i} \in Y^{c}$.

The quantisation process is different for each data set and explained in detail in Section IX. Taking the plasma beam data for example, as shown in Figure 8, the input space is the $2 \mathrm{D}$ coordinate-space around the edge of the plasma ball. This space is manually quantised into $N_{s}=11$ symbols/subregions, relating to the general locations the plasma beam can move to.

A conditional probability distribution is built using the training data that maps from the input space to pose space. The $r^{\text {th }}$ input symbol is mapped to the $q^{\text {th }}$ cut point cluster using $P\left(C_{q} \mid\right.$ input $\left._{r}\right)=p_{q, r}$ (where input $_{r} \in\{1, \ldots, N s\}$ ), which symbolises the probability of a cut point in cluster $q$ occurring when the user requests the $r^{t h}$ symbol.

Given that the $r^{t h}$ symbol captured a set $E_{r}$ of $N_{r}$ cut point samples, the mapping is computed as:

$$
p_{q, r}=\frac{P\left(\text { input }_{r}, C_{q}\right)}{P\left(\text { input }_{r}\right)}=\frac{\left|C_{q} \cap E_{r}\right|}{N_{r}}
$$

where $P\left(\right.$ input $\left._{r}\right)=\frac{N_{r}}{N_{c}}, P\left(\right.$ input $\left._{r}, C_{q}\right)=\frac{\left|C_{q} \cap E_{r}\right|}{N_{c}}$ and $\sum_{r} p_{q, r}=1$. This is used at run-time to weight the chosen cut points given a user selected input symbol (input). As a result, Equation 5 is altered to:

$$
\phi_{m}=P\left(C_{C_{t+1}, m}^{z} \mid C_{t}\right) \cdot P^{\prime}\left(\mathbf{z}_{C_{t+1}, m}^{c}\right) \cdot \omega
$$

where

$$
\omega= \begin{cases}P\left(C_{i} \mid \text { input }\right) & \text { if } \mathbf{y}_{i} \in \mathbf{Y}_{i}^{c} \\ 0 & \text { otherwise }\end{cases}
$$

\section{Animation/Results}

This Section presents the results of the MIMiC system demonstrated in three different data formats: motion capture, video, and conversation. ${ }^{1}$

\section{A. Experiments with Motion Captured Data}

Six motion capture sequences were projected down into their combined lower dimensional eigenspace using the approach detailed in Section V. This made up a data set of 2884 frames at a reduced 30 dimensions. The six individual motion sequences were of a 'male walk', 'female walk', 'drunk walk', 'skip', 'march', and 'run'. The sequences were captured from the same actor using 36 markers to cover the main joints of the human body. Using our unsupervised segmentation approach, as detailed in Section VII-A, $61 \mathrm{k}$-medoid points were defined, using $\theta=0.5$ to produce 228 subsequences. In the quantisation process, as explained in Section VIII-A, $N_{s}=6$, relating to the six different types of walks in the data set. Figure 9 shows the results of synthesis and blending between the different types of walks. MIMiC is demonstrated

\footnotetext{
${ }^{1}$ Supplementary downloadable material is also available demonstrating these results at http://ieeexplore.ieee.org. This material is $6.8 \mathrm{MB}$ in size. Audio is incorporated in the conversation demonstration so please use headphones or speakers.
}

by giving a user real time control over the type of walk to animate. In this example, the user chooses to animate from a female walk to a drunk walk, then to a male walk, march, run and skip. Frames $a, b, c, d, e$ and $f$ are cut points used to make smooth transitions from one type of walk to another. As suggested by the dotted red lines, these cut points can be used to transition to walks not demonstrated in this example. To improve blends between transitions of varying speeds, velocity interpolation is used to gradually speed up or slow down motion leading to and from a transition. Supplementary material is available, demonstrating the real-time animation of the generated motion sequence.

\section{B. Experiments with Video Data}

Two video sequences were recorded using a webcam. One was of a candle flame and the other of plasma beams from a plasma ball.

The candle flame sequence $(185 \times 140$ pixels, 15 frames per second) was 3:20 minutes long containing 3000 frames. The recording was of a candle flame performing 3 different motions, blowing left, blowing right and burning in a stationary position. The dimension reduction process, projected the data down to 42 dimensions. Using our unsupervised segmentation approach, $90 \mathrm{k}$-medoid points were defined, using $\theta=0.25$ to produce 309 subsequences. $N_{s}=3$ giving the user control over the three discrete states of the candle flame. As shown in Figure 10, using MIMiC, the user can control the three discrete states of the candle flame motion. If the animation is at a blow right state, it has to travel to the stationary state before a blow left state can be reached, expressed by the transition matrix and determined through dynamic programming. Using simple image processing to detect motion we allow the user to directly interact with the animation by using hand motion to simulate a breeze which effects the direction of the flame in animation. See supplementary material.

The plasma beam sequence was also captured with a webcam $(180 \times 180$ pixels, 15 frames per second). The recording was 3:19 minutes long containing 2985 frames. Dimension reduction projected this data set down to 100 dimensions, and the unsupervised segmentation algorithm defined $53 \mathrm{k}$ medoid points, using $\theta=0.6$ to produce 230 subsequences. The plasma beam sequence has more varying movement than the candle flame. It produces motion ranging from multiple random plasma beams to a concentrated beam from a point of contact anywhere around the edge of the ball. As a result, the modelled plasma beam offers more varying degrees of control. We divide the different states of the plasma beam motion round the edges of the plasma ball into eleven discrete states. Using a mouse cursor or touch screen, the user can control the movement of the plasma beam around the edges of the plasma ball, as shown in Figure 11(A). Supplementary material is available, demonstrating the real time rendering of the candle flame and plasma beam motions.

\section{Experiments with Conversation Data}

Two people conversing with each other were recorded using two SD (Standard Definition) cameras $(720 \times 576$ pixels, 25 


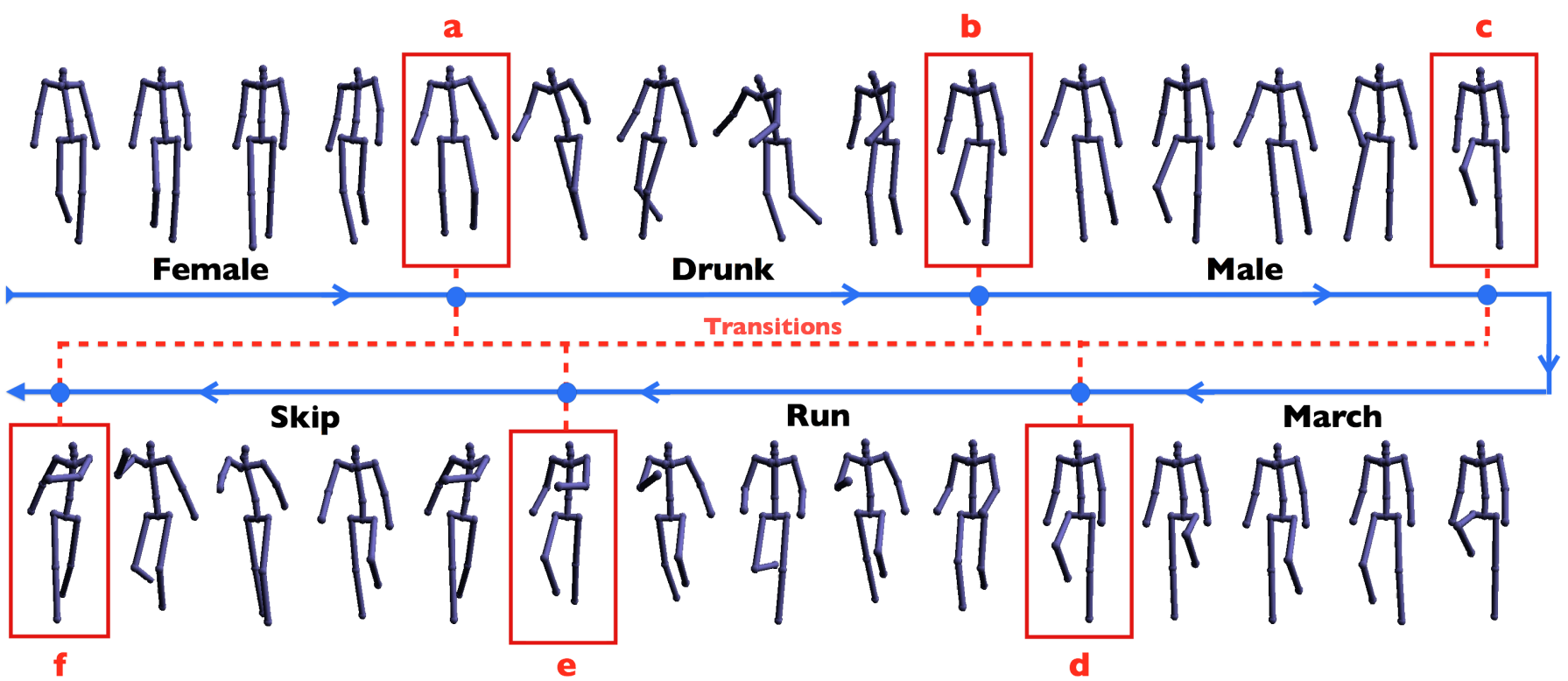

Fig. 9. Image showing synthesis and blending of different types of motion captured walks. The blue arrow indicates the motion trajectory of the motion synthesis. The frames in boxes $(a, b, c, d, e$ and $f)$ are cut points used for transitioning from one type of motion to another.

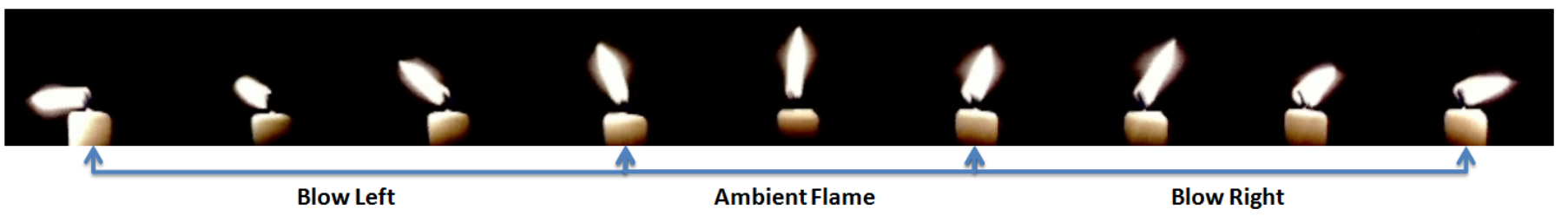

Fig. 10. Image showing candle flame synthesis. Using MIMiC, the user is able to control the three discrete states of the candle flame and blend between them. To transition from a flame blow left state to a blow right state, the system will perform a transition to a stationary flame state first resulting in a better looking transition

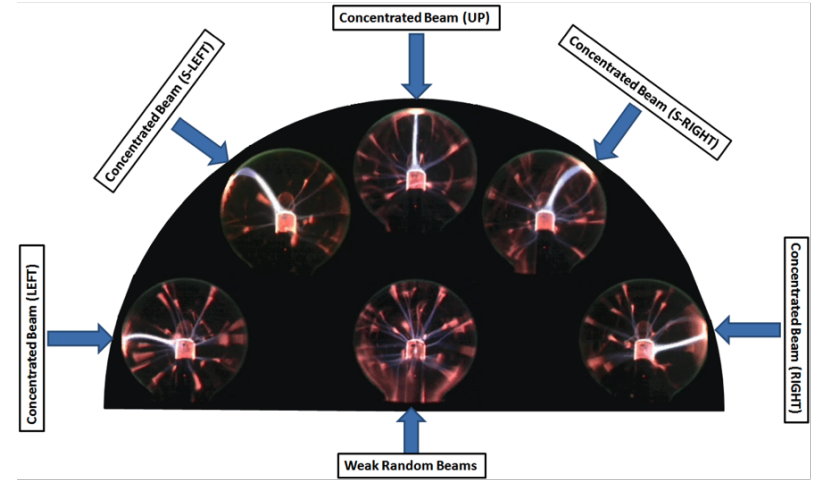

(A)

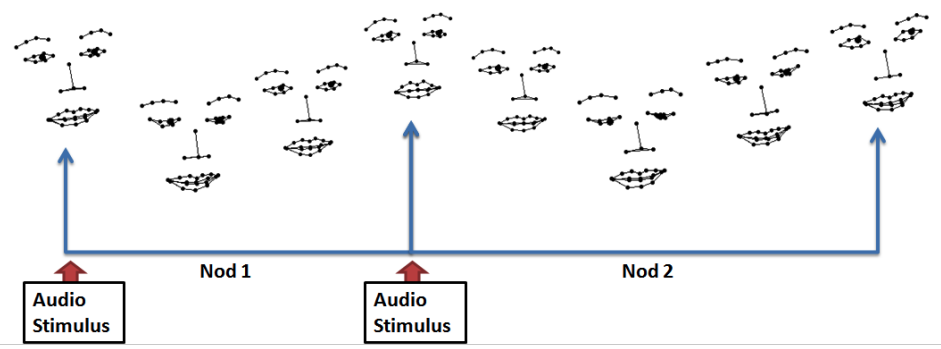

(B)

Fig. 11. (A) Image showing plasma beam synthesis. Arrows indicate direction of sythesis flow. (B) Image showing synthesis of a nod as a response to audio stimulus

frames per second) and a microphone $(48 \mathrm{kHz})$. They sat face to face at a comfortable distance apart. The frontal view of each face was captured whilst they conversed for 12 minutes. One of the subjects was male and the other female. They spoke in fluent english and considered themselves friends.

The data was analysed and one of the subjects was chosen to be the expressive listener whilst the other was deemed the speaker. Periods when the listener is clearly engaged in listening to the speaker with no co-occurring speech were extracted. This produced 10 audio-visual fragments which were combined to produce a total of 2:30 minutes of data.
The facial features of the listener, including head pose, were tracked using a Linear Predictor tracker [31]. 44 2D points were used to cover the contour of the face including the eye pupils. When processed, this produced $55 \mathrm{k}$-medoid points and 146 subsequences using $\theta=0.03$, which we reduced to 50 dimensions using PCA. As shown in Figure 11 (B), the movements of these $2 \mathrm{D}$ points are dynamically generated from MIMiC in real-time. The audio stimulus uses the conditional probability to derive various visual responses based on its content. The most prominent visual responses are head nods, although other expressions like smiles, eye brow lifts and 
TABLE I

SCORES FOR VISUAL RESPONSES TO AUDIO. COLUMN 1 IS THE NUMERICAL INDEX OF PEOPLE GIVING SCORES. COLUMN 2 AND 3 ARE THE NORMALISED AND AVERAGED SCORES FOR VISUAL RESPONSES THAT ARE AUDIO DRIVEN AND RANDOMLY PLAYING RESPECTFULLY. COLUMN 4 IS THE AUDIO DRIVEN SCORES DIVIDED BY THE RANDOM PLAY SCORES

\begin{tabular}{|l|c|c|c|}
\hline Person & Audio-Model & Random Play & $\frac{\text { Model }}{\text { Random }}$ \\
\hline 1 & 0.66 & 0.32 & $\mathbf{2 . 1}$ \\
\hline 2 & 0.56 & 0.43 & $\mathbf{1 . 3}$ \\
\hline 3 & 0.86 & 0.11 & $\mathbf{7 . 8}$ \\
\hline 4 & 0.45 & 0.56 & 0.8 \\
\hline 5 & 0.45 & 0.56 & 0.8 \\
\hline 6 & 0.9 & 0.1 & $\mathbf{9}$ \\
\hline 7 & 0.27 & 0.7 & 0.4 \\
\hline 8 & 0.78 & 0.18 & $\mathbf{4 . 3}$ \\
\hline 9 & 0.55 & 0.43 & $\mathbf{1 . 3}$ \\
\hline 10 & 0.78 & 0.2 & $\mathbf{3 . 9}$ \\
\hline 11 & 0.65 & 0.33 & $\mathbf{2}$ \\
\hline 12 & 0.7 & 0.3 & $\mathbf{2 . 3}$ \\
\hline 13 & 0.5 & 0.5 & $\mathbf{1}$ \\
\hline 14 & 0.57 & 0.42 & $\mathbf{1 . 3}$ \\
\hline \hline Aver. & 0.62 & 0.37 & \\
\hline Std. Dev. & 0.18 & 0.18 & \\
\hline
\end{tabular}

TABLE II

AVERAGE AND STANDARD DEVIATION OF SCORES FOR VISUAL RESPONSES TO AUDIO BASED ON REPLAY OF ORIGINAL DATA AND RANDOM PLAY

\begin{tabular}{|l|c|c|}
\hline Person & Replay & Random Play \\
\hline Aver. & 0.57 & 0.45 \\
\hline Std. Dev. & 0.3 & 0.33 \\
\hline
\end{tabular}

blinks are generated when appropriate.

The audio stream is represented using 12 MFCCs and a single energy feature of the standard HTK setup, a configuration commonly used in speech analysis and recognition [32]. The frame rate of 100 frames per second was selected with $50 \%$ overlap, i.e., the window size is $20 \mathrm{~ms}$ and the step size $10 \mathrm{~ms}$. $N_{s}=25$ symbols/classes of the speaker's MFCC is used as the input space. The extraction of these classes is automatic using the k-means algorithm. Here, $N_{s}$ is chosen experimentally to represent an even distribution of the MFCCs. The conditional probability, as explained in Section VIII-A, is then learnt that maps MFCC input features to pose space to map the audio features to the animation.

For testing, another set of audio sequences were captured from the same speaker in a casual conversation. 15 speech fragments were selected from the conversation totalling 2:31 minutes. Using the projection mapping from audio features to pose space, these speech fragments generated a synthetic listener with plausible visual responses. Supplementary material is available, demonstrating the results.

To validate results, 14 people were asked to listen to the 15 test audio segments and to score between 1 and 10 how well the visual model responded to the audio as a synthetic listener in the conversation. They were unaware that approximately half of the visual responses to the audio segments were playing randomly regardless of the audio input whilst the other half were generated from the audio input to the model. The results are listed in Table I. We normalised each person's score and took the average for both audio-model generation and random play. As shown in the fourth column of Table I entitled ' $\frac{\text { Model }}{\text { Random }}$ ', 11 out of 14 generated a score greater than or equal to 1 , showing preference to the visual responses generated by the audio input than by the random play. Although the majority could tell the difference, the margins of success are not considerably high producing an average of 0.62 . Several assumptions may be drawn from this. As nods are the most effective non-verbal response of an engaged listener, random nods may provide an acceptable response to a speaker. To try to validate these tests, the same 14 people were asked to repeat the test but this time on the 10 audio segments used in training the model. 5 out of 10 of the audio segments were randomly played visual responses and the other 5 were replays of the original audio-visual pairing. Results in Table II show that for a baseline test on ground truth data, where we know a direct correlation exists between the audio signal and the visual response, the participants provide very similar levels of scoring. This indicates that our animations are very nearly as convincing as a real listener in terms of the responses provided to audio data.

\section{CONCLusion}

$\mathrm{MIMiC}$ can generate novel motion sequences, giving a user real-time control. We show that the Motion Model can be applied to various motion formats such as 3D motion capture, video textures, and 2D tracked points. It can also produce novel sequences with the same realism inherent in the original data. We have demonstrated that the Multimodal Controller can provide interactive control, using a number of interfaces including audio. We have also shown that it is possible to learn a conversational cue model using MIMiC to derive appropriate responses using audio features.

For future work, a possible improvement to the Motion Model would be to derive a method of deducing $N_{c}$ and $\theta$ automatically. However, given the data set, the user will still need control in regulating the level of connectivity and quality of the animation which are governed by these parameters. Although $\theta$ provides a tolerance on how close cut points need to be to form valid transitions, this does not eliminate the risk of falsely identifying transition points. Such a risk is more prominent in the motion capture data set with regards to mirrored poses (during the crossing of the legs), whereby transitions to a mirror pose will result in unrealistic reverse motion. Though MIMiC incorporates temporal information using a $1^{\text {st }}$ order dynamics model, future work will explore the use of a $2^{\text {nd }}$ order dynamics to account for such ambiguities. Also, in cases where there is extremely large variable density across the data, a means of pruning the clusters based on similarity would prove valuable. Whilst increasing the number of k-medoids to account for high variable density, such an addition would limit the number of clusters in the high density areas whilst subsequently allowing the clusters in the lower density areas to increase, resulting in more evenly distributed points for transitions.

An additional improvement would be to incorporate contextual information into the conversation data set, such as topic of conversation and specific social signals like eye gaze, nodding and laughing. However, further study is needed to derive a social dynamics model between the speaker and listener to parameterise these exchanges in social behaviour. 


\section{REFERENCES}

[1] D. Heeger and J. Bergen, "Pyramid-based texture analysis," in Proc. of SIGGRAPH 95, August, Los Angeles, California, 1995, pp. 229-238.

[2] M. Szummer and R. Picard, "Temporal texture modeling," in In Proc. of IEEE Int. Conf. on Image Processing, 1996, 1996, pp. 823-826.

[3] A. Efros and T. Leung, "Texture synthesis by non-paramteric sampling," in In Int. Conf. on Computer Vision, 1999, pp. 1033-1038.

[4] L.-Y. Wei and M. Levoy, "Fast texture synthesis using tree-structure vector quantization," in Proc. of SIGGRAPH 2000, July, 2000, pp. 479488.

[5] V. Kwatra, A. Schodl, I. Essa, G. Turk, and A. Bobick, "Graphcut textures," in Proc. of SIGGRAPH 2003, 22, 3, 2003, pp. 277-286.

[6] K. Bhat, S. Seitz, J. Hodgins, and P. Khosla, "Flow-based video synthesis and editing," in Proc. of SIGGRAPH 2004, 2004.

[7] N. F. Troje, "Decomposing biological motion: A framework for analysis and synthesis of human gait patterns," J. Vis., vol. 2, no. 5, pp. 371-387, 92002.

[8] K. Pullen and C. Bregler, "Synthesis of cyclic motions with texture," 2002.

[9] D. Okwechime and R. Bowden, "A generative model for motion synthesis and blending using probability density estimation," in Fifth Conference on Articulated Motion and Deformable Objects, 9-11 July, Mallorca, Spain, 2008.

[10] J. Wang, D. Fleet, and A. Hertzmann, "Gaussian process dynamical models for human motion," IEEE transactions on pattern analysis and machine intelligence, vol. 30, no. 2, pp. 283-298, 2008

[11] L. M. Tanco and A. Hilton, "Realistic synthesis of novel human movements from a database of motion captured examples," In Proceedings of the IEE Workshop on Human Motion HUMO 2000), 2000.

[12] H. Rachel and M. Gleicher, "Parametric motion graph," 24th Int. Symposium on Interactive 3D Graphics and Games, pp. 129-136, 2007.

[13] H. Shin and H. Oh, "Fat graphs: Constructing an interactive character with continuous controls," in Proceedings of the 2006 ACM SIGGRAPH/Eurographics symposium on Computer animation, 2006, p. 298.

[14] K. Balci and L. Akarun, "Generating motion graphs from clusters of individual poses," 24th Int. Symposium on Computer and Information Sciences, pp. 436-441, 2009.

[15] P. Beaudoin, S. Coros, M. van de Panne, and P. Poulin, "Motionmotif graphs," in In Proc. of the 2008 ACM SIGGRAPH/Eurographics Symposium on Computer Animation, 2008, pp. 117-126.

[16] L. Kovar, M. Gleicher, and F. Pighin, "Motion graphs," In Proceedings of ACM SIGGRAPH, 21, 3, Jul, pp. 473-482, 2002.

[17] O. Arikan, D. Forsyth, and J. O'Brien, "Motion synthesis from annotation," In ACM Transaction on Graphics, 22, 3, July, (SIGGRAPH 2003), pp. 402-408, 2003.

[18] A. Treuille, Y. Lee, and Z. Popovic, "Near-optimal character animation with continuous control," in Proc. of SIGGRAPH 2007 26(3), 2007.

[19] A. Schödl, R. Szeliski, D. Salesin, and I. Essa, "Video textures," in Proceedings of the 27th annual conference on Computer graphics and interactive techniques, SIGGRAPH 2000. ACM Press/Addison-Wesley Publishing Co. New York, NY, USA, 2000, pp. 489-498.

[20] M. Flagg, A. Nakazawa, Q. Zhang, S. Kang, Y. Ryu, I. Essa, and J. Rehg, "Human video textures," in Proceedings of the 2009 symposium on Interactive 3D graphics and games. ACM, 2009, pp. 199-206.

[21] J. Lee, J. Chai, P. Reitsma, J. Hodgins, and N. Pollard, "Interactive control of avatars animated with human motion data," ACM Transactions on Graphics, vol. 21, no. 3, pp. 491-500, 2002.

[22] C. Bregler, M. Covell, and M. Slaney, "Video rewrite: Driving visual speech with audio," in Proceedings of the 24th annual conference on Computer graphics and interactive techniques. ACM Press/AddisonWesley Publishing Co. New York, NY, USA, 1997, pp. 353-360.

[23] M. Brand, "Voice puppetry," in Proceedings of the 27th annual conference on Computer graphics and interactive techniques, SIGGRAPH 1999. ACM Press/Addison-Wesley Publishing Co. New York, NY, USA, 1999, pp. 21-28.

[24] M. Stone, D. DeCarlo, I. Oh, C. Rodriguez, A. Stere, A. Lees, and C. Bregler, "Speaking with hands: Creating animated conversational characters from recordings of human performance," in ACM Transactions on Graphics (TOG), SIGGRAPH 2004, vol. 23, no. 3. ACM New York, NY, USA, 2004, pp. 506-513.

[25] T. Jebara and A. Pentland, "Action reaction learning: Analysis and synthesis of human behaviour," in Workshop on the Interpretation of Visual Motion - Computer Vision and Pattern Recognition Conf., 1998.

[26] D. Okwechime, E. J. Ong, and R. Bowden, "Real-time motion control using pose space probability density estimation," in IEEE Int. Workshop on Human-Computer Interaction, 2009.
[27] E. Sahouria and A.Zakhor, "Content analysis of video using principal components," in IEEE Trans. on Circuits and Systems for Video Technology, vol. 9, no. 8, 1999.

[28] M. Alexa and W. Muller, "Representing animations by principal components," in Computer Graphics Forum, 19(3), 2000, pp. 411-418.

[29] A. Moore, "A tutorial on kd-trees," Extract from PhD Thesis, 1991, available from http://www.cs.cmu.edu/simawm/papers.html.

[30] W. Hastings, "Monte Carlo sampling methods using Markov chains and their applications," Biometrikal, vol. 57, no. 1, pp. 97-109, 1970.

[31] E. J. Ong, Y. Lan, B. J. Theobald, R. Harvey, and R. Bowden, "Robust facial feature tracking using selected multi-resolution linear predictors," in Int. Conf. Computer Vision ICCV 2009, 2009.

[32] A. Mertins and J. Rademacher, "Frequency-warping invariant features for automatic speech recognition," in 2006 IEEE Int. Conf. on Acoustics, Speech and Signal Processing, 2006. ICASSP 2006 Proc., vol. 5, 2006.

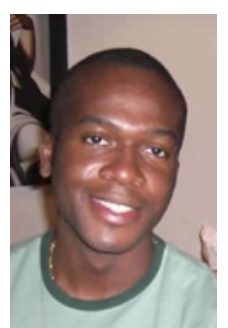

Dumebi Okwechime Dumebi Okwechime received the MEng degree in Electrical and Electronic Engineering from the University of Surrey, United Kingdom in 2006 . He is currently a $\mathrm{PhD}$ candidate in the Centre for Vision Speech and Signal Processing at the University of Surrey. His research interests include real-time dynamic models, human computer interaction and interfaces, and multimodal conversational agents.

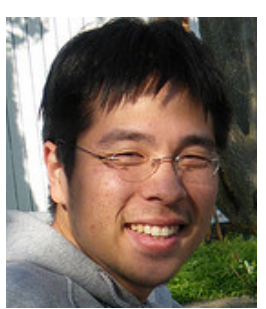

Eng-Jon Ong Eng-Jon Ong received a computer science degree in 1997 and the $\mathrm{PhD}$ degree in computer vision in 2001 from Queen Mary, University of London. Following that, he joined the Center for Vision, Speech and Signal Processing at the University of Surrey as a researcher. His main interests are in visual feature tracking, data mining, pattern recognition and machine learning methods.

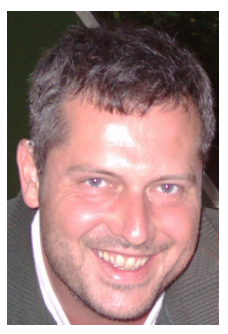

Richard Bowden Richard Bowden received a BSc degree in Computer Science from the University of London in 1993, a MSc in 1995 from the University of Leeds and a PhD in Computer Vision from Brunel University in 1999 . He is currently a Reader at the University of Surrey, UK where he leads the Cognitive Vision Group within the Centre for Vision Speech and Signal Processing. His research centres on the use of computer vision to locate, track and understand humans. His research into tracking and artificial life received worldwide media coverage, appeared at the British Science Museum and the Minnesota Science Museum. He has won a number of awards including paper prizes for his work on sign language recognition, as well as the Sullivan Doctoral Thesis Prize in 2000 for the best UK PhD thesis in vision. He was a member of the British Machine Vision Association (BMVA) executive committee and company director for 7 years. He is a London Technology Network Business Fellow, a member of the British Machine Vision Association, a Fellow of the Higher Education Academy and a Senior Member of the Institute of Electrical and Electronic Engineers. 